\title{
Right but basically wrong: Comments on Canales et al., 'A Critical Assessment of the Consciousness by Synchrony Hypothesis'
}

\section{EUGENIO RODRÍGUEZ ${ }^{1}$ and JOSÉ CORTÉS ${ }^{1}$}

${ }^{1}$ Escuela de Psicología, Pontificia Universidad Católica de Chile

The authors are right in holding the view that neural synchrony does not seem to provide a solution for the 'hard problem of consciousness' (Chalmers 1996). They are also right in that most of the evidence presented for the role of neural synchronization in perceptual binding is correlative and not causal. They are essentially wrong in all their remaining points.

It is true that neural synchrony does not solve the hard problem, but then, nobody has proposed a solution for the hard problem. It is not even clear that such a problem exists or that a scientific solution can ever be given to the hard problem (Dennett 1991). Certainly it is premature to make a definitive statement on this subject at the present stage of research.

By contrast, neural synchrony does seem to be at least part of the neural correlates of consciousness, which ask what are the minimal neural activities and structures that are necessary and sufficient for a conscious act to take place (Crick \& Koch, 1990). In a recent paper we have been able to show that, long-range synchrony correlates with visual awareness but local synchrony does not (Melloni et al. 2007). This result, suggests that synchronic activity inside a restricted cortical area remains unconscious and that consciousness arises only when 'all the brain' is informed via long-range synchronic interactions. It can be hypothesized that this would be the basis for the unified character of experiential consciousness.

The authors correctly point to the correlative nature of most of the evidence supporting a role of neural synchrony both in consciousness and in visual feature binding. To our knowledge there is a single experiment showing the causal involvement of neural synchrony in an odor discrimination task (Stopfer et al. 1997). However it must be stated that the same holds true for all electrophysiology in all the fields of neuroscience. From the studies of Hubel and Wiesel on, essentially all electrophysiology consists of manipulating the stimuli, recording the neural response and proposing mechanisms based on the correlations found. The only alternative theory for visual binding (the cardinal cell theory, Barlow 1972) is also mostly based on correlative evidence with the only exception of a few works conducted by Shadlen and collaborators (Huk \& Shadlen 2005)

An argument is made against synchrony as a mechanism for consciousness because of the participation of synchrony in nonconscious (anesthetized) animals and in brain areas not obviously related to perceptual consciousness (the hippocampus). Both arguments are logically false. The fallacy is tantamount to claiming that because 'John' has been shown to be a mammal then it cannot be a human. If they were true one should conclude that sugar metabolism doesn't participate in brain function because it has been shown to be present in organisms without a brain and in body parts that do no participate in brain function.

A point is made against neural synchrony participation in visual binding because in some of the earliest experiments, the consciousness status of the animal is not known. In fact Gray and Singer's cat was anesthetized (as also was Hubel and Wiesel's monkey, whose work in vision is 
not contested) because they were working on a very basic level of visual binding which, like contour integration phenomenon, and other gestalt grouping laws, are supposed to operate in a preattentive and preconscious way as proposed by Helmholtz and the gestalt psychologists. Thus, the consciousness status of the subjects is irrelevant at this level of visual binding. In contrast, there is a number of human studies showing that neural synchrony does indeed correlate with the visual binding of stimuli (Tallon-Baudry et al. 1997, Rodriguez et al. 1999, for reviews see Tallon-Baudry \& Bertrand 1999, Singer 1999)

The authors also suggest that neural synchrony which seems to happen in bursts could not possibly be the basis for 'the continuous flow' which characterizes consciousness. In the first place it is as yet not clear whether perception is really continuous or discrete (VanRullen and Koch 2003) but even if it were continuous, neural synchrony could still underlie its operation either by a smoothing based on perceptual memory, either because it is possible that synchrony is in fact a continuous process that cannot be recovered because its spatial distribution diverges a few hundred milliseconds after stimulus presentation and thus does not add-up on averages. Of course these are just conjectures but show that synchrony cannot be ruled out to explain the continuous flow of consciousness.

The final remarks of the authors are particularly interesting and particularly misleading. "who coordinates the synchronized activity?' Answer: nobody, there is nobody there to coordinate anything. "Which neuronal assemblies would be "in charge" to interpret that coordination?' Answer: No one is in charge. The coordination is the interpretation. 'How could neurons distinguish neuronal inputs as belonging either to local or to large-scale interactions?' Answer: They cannot and don't care. It is the complete network and its behavior who stand for the distinction. 'Would we need an additional neuronal mechanism to specify those distinctions?' Answer: No, such additional mechanism would be a homunculus. We believe that, in order to escape homuncular explanations the only possibility is that, the large-scale neural synchronic pattern (or whatever the correlate for consciousness is), must be both the audience and the spectacle in the Cartesian Theater of Mind.

\section{REFERENCES}

BARLOW H B (1972) Single units and sensation: A neuron doctrine for perceptual psychology? Perception 1: 371394

CHALMERS D (1996) The conscious mind: In search of a fundamental theory. Oxford: Oxford University Press

CRICK F and KOCH C (1990) Towards a neurobiological theory of consciousness. Seminars in Neuroscience 2: 263-75

DENNETT DC (1991) Consciousness Explained. Boston, MA, and London, Little, Brown and Co

HUK AC, SHADLEN MN (2005) Neural activity in macaque parietal cortex reflects temporal integration of visual motion signals during perceptual decision making. J Neurosci 25: 10420-36

MELLONI L, MOLINA C, PENA M, TORRES D, SINGER W, RODRIGUEZ E (2007) Synchronization of neural activity across cortical areas correlates with conscious perception. J Neurosci 27: 2858-65

SINGER W (1999) Neuronal synchrony: a versatile code for the definition of relations? Neuron 24: 49-65, 11125

STOPFER M, BHAGAVAN S, SMITH BH, LAURENT G (1997) Impaired odour discrimination on desynchronization of odour-encoding neural assemblies. Nature 390: 70-4

TALLON-BAUDRY C, BERTRAND O, DELPUECH C, PERMIER J (1997) Oscillatory gamma-band (30-70 $\mathrm{Hz}$ ) activity induced by a visual search task in humans. J Neurosci 17: 722-34

TALLON-BAUDRY C, BERTRAND O (1999) Oscillatory gamma activity in humans and its role in object representation. Trends Cogn Sci 3: 151-162

VANRULLEN R, KOCH C (2003) Is perception discrete or continuous? Trends Cogn Sci 7: 207-213 